\title{
The prognostic significance of BMI1 expression in invasive breast cancer is dependent on its molecular subtypes
}

\author{
Maryam Althobiti ${ }^{1,2} \cdot$ Abir A. Muftah ${ }^{3} \cdot$ Mohammed A. Aleskandarany ${ }^{1} \cdot$ Chitra Joseph $^{1} \cdot$ Michael S. Toss $^{1}$. \\ Andrew Green ${ }^{1} \cdot$ Emad Rakha $^{1,4}$
}

Received: 27 December 2019 / Accepted: 1 June 2020 / Published online: 10 June 2020

(c) The Author(s) 2020

\begin{abstract}
Purpose BMI1, which is a major component of the polycomb group complex 1, is an essential epigenetic repressor of multiple regulatory genes and has been identified as a cancer stem cell (CSC) marker in several cancers. However, its role in breast cancer (BC) remains to be defined. In this study, we have evaluated the prognostic significance of BMI1 among the different molecular subtypes and assessed its association with other breast CSC markers (BCSC).

Material and method BMII copy number and mRNA was assessed in large and well-characterised cohorts of early-stage BC patients [METABRIC $(n=1980)$ and the Bc-GenExMiner $(n=9616)$ databases]. BMI1 protein expression was assessed using tissue microarray and immunohistochemistry in a cohort of 870 invasive BC patients with long-term outcome data and the expression of a panel of BCSC markers was monitored.

Result BMI1 expression, prognostic significance and its association with BCSC markers were differed between molecular classes. In the luminal oestrogen receptor-positive (ER+) BC, BMI1 showed significantly higher expression compared to ER-tumours. BMI1 showed positive correlation with favourable prognostic features and it was negatively associated with the expression of key BCSC markers (ALDH1A1, CD24, CD44, CD133, SOX10 and SOX9). High expression of BMI1 was associated with longer breast cancer-specific survival (BCSS) independent of other prognostic variables. In the basal triple negative BC subtype, BMI1 expression showed positive association with CD133 and SOX10 and it was significantly associated with shorter BCSS.

Conclusion High BMI1 expression is associated with clinicopathological variables and outcome in BC. However, this association is dependent on the molecular subtypes. Further functional assessment to detect its underlying mechanistic roles in $\mathrm{BC}$ subtypes is warranted.
\end{abstract}

Keywords BMI1 $\cdot$ Breast cancer $\cdot$ Oestrogen receptor positive $\cdot$ Outcome $\cdot$ Breast cancer stem markers

\section{Introduction}

Maryam Althobiti and Abir A. Muftah are Joined first authors.

Electronic supplementary material The online version of this article (https://doi.org/10.1007/s10549-020-05719-x) contains supplementary material, which is available to authorized users.

Emad Rakha

emad.rakha@nottingham.ac.uk; Emad.rakha@nuh.nhs.uk

1 Division of Cancer and Stem Cells, Nottingham Breast Cancer Research Centre, University of Nottingham Biodiscovery Institute, University Park, Nottingham NG7 2RD, UK

2 Department of Clinical Laboratory Science, College of Applied Medical Science, Shaqra University 33, Shaqra 11961, Saudi Arabia
Polycomb complex protein or B-lymphoma Moloney murine leukaemia virus insertion region-1 (BMI1) is a member of the polycomb family which are a group of transcriptional

3 Department of Pathology, Faculty of Medicine, Faculty of Medicine and Health Science, University of Benghazi, Benghazi, Libya

4 Department of Histopathology, Nottingham University Hospital NHS Trust, City Hospital Campus, Hucknall Road, Nottingham NG5 1PB, UK 
repressors [1, 2]. BMI1 has a RING finger at the N-terminus, a central helix-turn-helix domain and a carboxyl-terminal PEST-like domain at the C-terminal end. The RING domain is required for BMI1 to localise to DNA strand breaks; therefore, it is involved in DNA damage response. The central helix-turn-helix domain with RING domain increases the life span of the cell. PEST domain is required for protein degradation [3]. BMI1 has been reported to be involved in several different pathways such as Wnt, Akt, Notch and Hedgehog signalling [4-7]. Hence, BMI1 has been shown to behave as a key regulator in the self-renewal, differentiation and tumour initiation of breast cancer stem cells (BCSC) [8]. In in vitro models, it has been observed that overexpression of BMI1 is linked to increased cell motility and invasion in BC $[9,10]$. Arnes et al. have reported that low expression of BMI1 is associated with high expression of Aldehyde dehydrogenase 1 (ALDH1) in African BC patients, where ALDH1 has been used as a functional marker to define the BCSC [11]. BMI1 has also been considered as a poor prognostic and predictive biomarker in several types of cancer $[1,12,13]$. However, conflicting data have been reported in the same study where high expression of BMI1 at mRNA and protein levels was associated with high expression of ER and positive axillary lymph node metastasis, leading to some difficulty in the interpretation of the results [14].

There is little available evidence on the immunohistochemical expression of BMI1 in BC tissue samples and whether it is also considered a biomarker of poor or good prognosis in $\mathrm{BC}$. This study aimed to investigate the clinical and pathological relevance of BMI1 expression, including its genomic, transcriptomic and protein levels, in BC utilising large cohorts of early-stage $\mathrm{BC}$ with a long-term follow-up. This is to characterise the variation of BMI1 expression in different $\mathrm{BC}$ molecular subtypes and to explore the associations between BMI1 and a panel of relevant BCSC markers at both the mRNA and protein levels.

\section{Material and methods}

\section{BMI1 protein expression}

The study cohort comprised 870 invasive BCs derived from the retrospective Nottingham Primary Breast Carcinoma Series of patients presenting to Nottingham City Hospital between 1986 and 1998. Patients' clinical and pathological data including age at diagnosis, histological tumour type, tumour size, lymph node status, Nottingham Prognostic Index (NPI), lympho-vascular invasion (LVI) and adjuvant therapy were available and prospectively maintained. Data for oestrogen receptor (ER), progesterone receptor (PgR), HER2 status and Ki67 data were available [15, 16]. ER and PgR cut-off values were defined as $\geq 1 \%$ and HER 2 status was defined as previously published $[15,17,18]$. Survival data were accessible and prospectively maintained including the following: (1) BC-specific survival (BCSS), defined as the time (in months) from the date of the primary surgical treatment to the time of death from breast cancer, and (2) distant metastasis free survival (DMFS), defined as the time (in months) from the surgery until the first event of distant metastasis [19]. The clinicopathological parameters for the study cohort are summarised in supplementary Table 1. BC intrinsic molecular subtypes were determined as previously descried [20]. Immunohistochemical detection of a panel of BCSC, including ALDH1A1, CD133, CD24, CD44, EPCAM, SOX9 and SOX10, had been previously performed [21-23] and these were used in the current study to assess their relationship with BMI1 expression.

\section{Tissue microarrays (TMAs) and immunohistochemical (IHC) evaluations}

Full face BC tissue sections were stained using IHC to evaluate the pattern of immunohistochemical BMI1 expression prior to staining of TMAs. Kidney tissue was used as a positive control while the negative control was obtained by omitting the application of primary antibody in the IHC staining protocol.

Formalin-fixed paraffin-embedded BC tissue samples were arrayed as previously described [24]. Prior to IHC staining, the specificity of the anti-BMI1 antibody was validated using Western blotting in MCF7, MDA-MB-231, SKBR3, MDA-MB-468 BC cell lines' lysates (American type culture collection, Rockville, MD, USA) and HeLa cells as control. This was performed using 1:5000 dilution of the primary antibody (EPR3745 (2), Abcam, UK), and 1:15,000 of the horseradish peroxidase-labelled secondary anti-rabbit antibody, with b-actin (1:5000) used as a loading control. A single band for BMI1 was observed at the predicted size $(40 \mathrm{kDa})$, which confirmed the specificity of the antibody (Supplementary Fig. 1). IHC staining was performed on 4 $\mu \mathrm{m}$ TMA sections using Novolink polymer detection system (Leica, Newcastle, UK). In brief, the antigen retrieval was performed in citrate buffer ( $\mathrm{pH}$ 6) in a microwave (Whirlpool JT359 Jet Chef $1000 \mathrm{~W}$ ) for $20 \mathrm{~min}$. The optimal dilution of BMI1 antibody in IHC was 1:100 and incubated for $1 \mathrm{~h}$ at room temperature. Stained TMA slides were scanned with high-resolution digital images (NanoZoomer; Hamamatsu Photonics, Welwyn Garden City, UK), at $20 \times$ magnification and viewed by Xplore viewer (Philips, Belfast UK). The BMI1 staining TMA cores were evaluated on the basis of a semiquantitative scoring of core digital images using a modified histochemical score ( $H$-score) [25]. All cases were scored by M. Althobiti, blinded to histopathological data and patients' outcome. To validate the results and test for the inter-observers reproducibility of the scoring, $10 \%$ of 
the cases were randomly selected and rescored by another observer (M. Toss).

\section{Genomic and transcriptomic analysis}

A cohort of $1980 \mathrm{BC}$ patients was evaluated in terms of BMII gene copy number $(\mathrm{CN})$ aberrations and mRNA expression using the Molecular Taxonomy of Breast Cancer International Consortium (METABRIC) [26, 27]. The cut-off point of BMII was determined using X-tile software (version 3.6.1, Yale University, USA), which was based on prediction of BCSS. The clinicopathological parameters of METABRIC series are summarised in supplementary Table 1 . There was no difference in the distribution

Table 1 The association of BMI1 and clinicopathological parameters in breast cancer in (Protein and mRNA levels)

\begin{tabular}{|c|c|c|c|c|c|c|}
\hline \multirow[t]{2}{*}{ Parameters } & \multicolumn{3}{|c|}{ Protein expression $(n=870)$} & \multicolumn{3}{|c|}{ mRNA expression (1980) } \\
\hline & $\begin{array}{l}\text { Low BMI1 } \\
\text { No }(\%)\end{array}$ & $\begin{array}{l}\text { High BMI1 } \\
\text { No }(\%)\end{array}$ & $\begin{array}{l}\mathrm{x}^{2} \\
P \text { value }\end{array}$ & $\begin{array}{l}\text { Low BMI1 } \\
\text { No. }(\%)\end{array}$ & $\begin{array}{l}\text { High BMI1 } \\
\text { No. }(\%)\end{array}$ & $\begin{array}{l}\mathrm{x}^{2} \\
P \text { value }\end{array}$ \\
\hline \multicolumn{7}{|l|}{ Patient age (years) } \\
\hline$<50$ & $451(80)$ & $111(20)$ & 0.22 & $147(37)$ & $249(63)$ & 11.809 \\
\hline$\geq 50$ & $243(79)$ & $65(21)$ & 0.635 & $411(28)$ & $1047(72)$ & 0.001 \\
\hline \multicolumn{7}{|l|}{ Tumour size $(\mathrm{cm})$} \\
\hline$<2$ & $369(82)$ & $83(18)$ & 1.77 & $193(31)$ & 429 (69) & 4.218 \\
\hline$\geq 2$ & $319(78)$ & $90(22)$ & 0.106 & $476(36)$ & $855(64)$ & 0.040 \\
\hline \multicolumn{7}{|l|}{ Tumour grade } \\
\hline Grade I & $90(69)$ & $40(31)$ & & $20(12)$ & $143(88)$ & \\
\hline Grade II & $211(75)$ & $69(25)$ & 22.33 & $162(22)$ & $567(78)$ & 91.336 \\
\hline Grade III & $385(86)$ & $64(14)$ & $<0.0001$ & $357(41)$ & $523(59)$ & $<0.0001$ \\
\hline \multicolumn{7}{|l|}{ Tubules formation } \\
\hline Score 1 & $26(67)$ & $13(33)$ & & & & \\
\hline Score 2 & $219(76)$ & $70(24)$ & 11.02 & $\mathrm{~N} \backslash \mathrm{A}$ & & \\
\hline Score 3 & $420(83)$ & $84(17)$ & 0.004 & & & \\
\hline \multicolumn{7}{|l|}{ Mitotic count } \\
\hline Score 1 & $187(73)$ & $68(27)$ & & & & \\
\hline Score 2 & $131(77)$ & $39(23)$ & 14.99 & $\mathrm{~N} \backslash \mathrm{A}$ & & \\
\hline Score 3 & $347(85)$ & $60(15)$ & $<0.0001$ & & & \\
\hline \multicolumn{7}{|l|}{ Nuclear pleomorphism } \\
\hline Score 1 & $11(78.6)$ & $3(21)$ & & & & \\
\hline Score 2 & $223(72.2)$ & $86(28)$ & 18.76 & & & \\
\hline Score 3 & $431(84.7)$ & $78(15)$ & $<0.0001$ & $\mathrm{~N} \backslash \mathrm{A}$ & & \\
\hline \multicolumn{7}{|l|}{ Axillary nodal stage } \\
\hline Stage I & $426(80)$ & $103(20)$ & 0.65 & 281(29) & $683(71)$ & \\
\hline Stage II & $214(79)$ & $57(21)$ & 0.721 & $29.4(29)$ & $414(71)$ & 3.418 \\
\hline Stage III & $46(77)$ & $14(23)$ & & $34.5(34)$ & $199(66)$ & 0.181 \\
\hline \multicolumn{7}{|l|}{ Nottingham prognostic index } \\
\hline Poor prognostic group & $119(83.8)$ & $23(16.2)$ & & $135(21)$ & $508(79)$ & \\
\hline Moderate prognostic group & $389(81.7)$ & $87(18.3)$ & 7.46 & $346(34)$ & $676(66)$ & 42.356 \\
\hline Good prognostic group & $180(74.1)$ & $63(25.9)$ & 0.024 & $77(41)$ & $112(59)$ & $<0.0001$ \\
\hline \multicolumn{7}{|l|}{ Oestrogen receptor } \\
\hline Negative & 213(91) & $20(9)$ & 26.12 & $224(56)$ & $177(44)$ & 165.226 \\
\hline Positive & $474(76)$ & $152(24)$ & $<0.0001$ & $319(23)$ & $1095(77)$ & $<0.0001$ \\
\hline \multicolumn{7}{|l|}{ Progesterone receptor } \\
\hline Negative & $309(87)$ & $48(13)$ & 14.48 & $368(42)$ & $502(58)$ & 116.00 \\
\hline Positive & $361(76)$ & $114(24)$ & $<0.0001$ & 190(19) & 794(81) & $<0.0001$ \\
\hline \multicolumn{7}{|l|}{ HER2 status } \\
\hline Negative & 557 (79) & $151(21)$ & 8.46 & 467 (29) & $1157(71)$ & 10.616 \\
\hline Positive & $103(90)$ & $11(10)$ & 0.002 & 90 (39) & $139(6)$ & 0.001 \\
\hline
\end{tabular}

Bold represents the significant $P$ values 
of clinicopathological parameters between Nottingham series and METABRIC series of patients (correlation coefficients $=0.733$, all $P<0.0001)$ [28].

To validate the prognostic significance of BMII mRNA expression, another publically available database (Breast Cancer Gene Expression Miner v4.0 (Bc-GenExMiner v4.0), with the online dataset available at https://bcgenex.centr egauducheau.fr), was used. This large dataset $(n=9616)$ allowed the evaluation of the prognostic role of BMII in $\mathrm{BC}$ cohorts, which include key prognostic parameters such as patients' age, tumour grade, nodal status, NPI, ER and molecular subtypes. Univariate analyses for molecular BC subtypes were performed [29].

\section{Statistical analysis}

IBM SPSS 24.0 (Chicago, IL, USA) software was used for statistical analysis. The inter-observer agreement was determined using intra-class correlation coefficient. BMI1 expression was categorised using 9.1 and $130 \mathrm{H}$-score cutoff of transcriptomic and IHC expression, respectively. Both cut-offs were determined using x-tile Bioinformatics software version 3.6.1 (Yale University, USA). The association between the categorical groups of BMI1 and clinicopathological parameters was analysed using a Chi-square test. The correlation of BMI1 and other biomarkers was tested using continuous data and Spearman test for the IHC analysis while the person test used for mRNA expression data. Associations with patient outcome were assessed using the Kaplan-Meier survival curves and the log-rank test. Cox proportional hazards regression models were built for multivariate survival analyses to estimate the hazard ratio (HR) of BMI1 adjusted by other well-known prognostic factors. A $P$ value of less than 0.05 (two- tailed) was considered significant in all statistical tests.

\section{Results}

\section{BMI1 expression in BC}

The IHC staining showed a homogenous staining pattern, with BMI1 expression localised in the nuclei of the invasive tumour cells. The staining intensities varied from negative (no stain) to strong intensity (Figs. 1, 2). Inter-observer agreement was determined, and the interclass correlation coefficient was 0.931 , indicating an excellent concordance between the 2 scorers.

In the whole $\mathrm{BC}$ cohort, the IHC expression of BMI1 ranged from 0 to $270 \mathrm{H}$-score. The data showed that high/ positive BMI1 IHC expression (H-score $>130$ ) was observed in $20 \%$ of cases $(176 / 870)$, whereas $80 \%$ of cases $(694 / 870)$ was considered low/negative. Interestingly, the immunoexpression of BMI1 in the luminal ER-positive $(E R+)$ subtype was higher than in ER-negative (ER-) subtypes $(P<0.0001)$. In the METABRIC cohort, high expression of BMI1 mRNA was seen in $65 \%$ of cases (1288/1980). In the Nottingham cases of the METABRIC cohort $(n=340)$, there was a strong association between $B M I I$ mRNA expression and protein expression $(P=0.001) . B M I I \mathrm{CN}$ gain was observed in $6 \%$ of cases $(113 / 1980)$ whereas $1 \%$ of cases (25/1980) showed CN loss. Supplementary Table 2 summarises the mean, median and the range of expression of BMI1 in $\mathrm{BC}$ subtypes at both protein and mRNA levels.
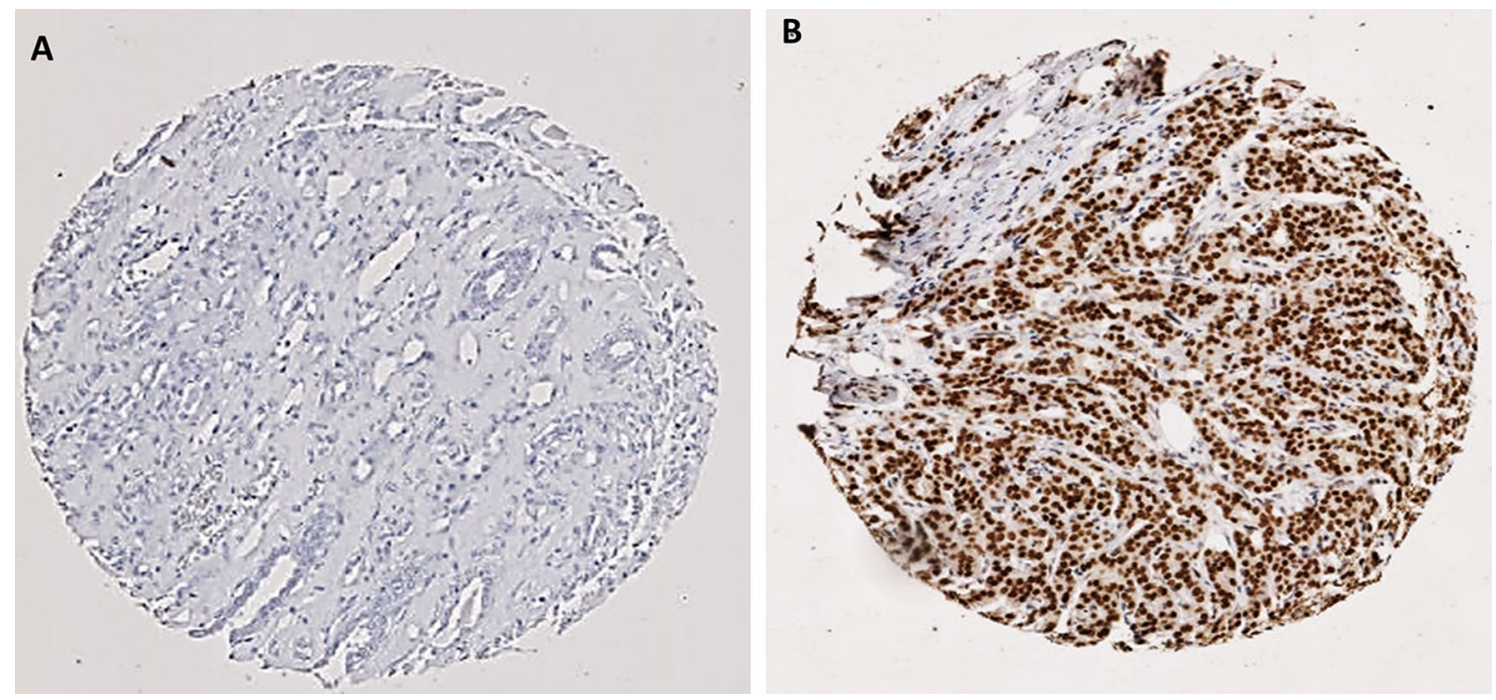

Fig. 1 Representative photomicrographs of the expression of BMI1 in invasive breast cancer a negative immunohistochemical (IHC) expression. b Positive IHC expression 

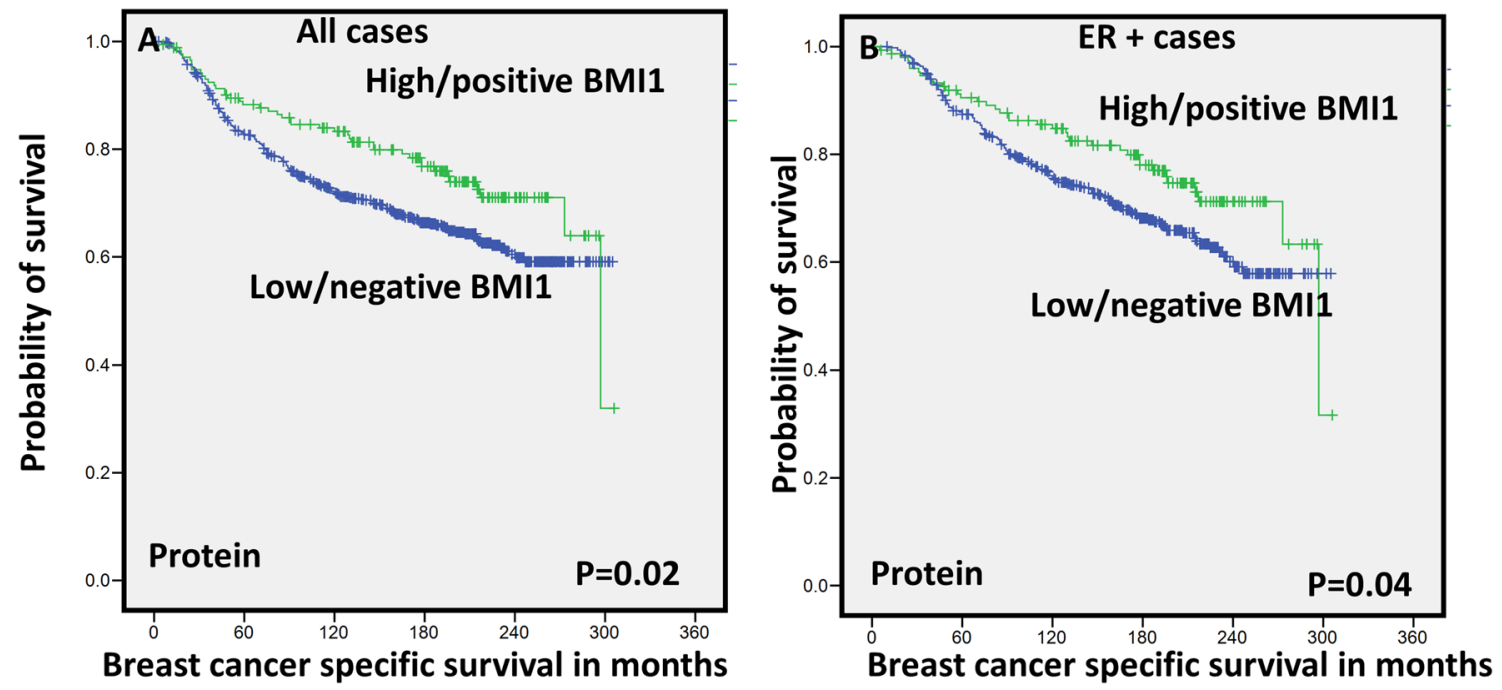

Fig. 2 Kaplan Meier survival plots for BMI1 expression (Protein) a breast cancer-specific survival (BCSS) in all cases and b breast cancerspecific survival (BCSS) in ER + cases

Table 2 Multivariate Cox regression hazard model including other prognostic clinicopathological parameters shows that high BMI1 (immunohistochemically) provided an independent prognostic value, associated with longer breast cancer-specific survival in the whole cohort

\begin{tabular}{llllr}
\hline Variable & Hazard ratio & \multicolumn{2}{l}{$\begin{array}{l}\text { 95\% Confidence } \\
\text { interval }(\mathrm{CI})\end{array}$} & $p$ value \\
\cline { 3 - 4 } & & Lower & Upper & \\
\hline Patient age & 0.88 & 0.69 & 1.135 & 0.346 \\
Tumour Grade & 1.61 & 1.32 & 1.96 & $<\mathbf{0 . 0 0 0 1}$ \\
Node stage & 1.99 & 1.67 & 2.38 & $\mathbf{< 0 . 0 0 0 1}$ \\
Tumour size & 0.67 & 0.52 & 0.87 & $\mathbf{0 . 0 0 2}$ \\
Bmi1 & 0.72 & 0.51 & 0.99 & $\mathbf{0 . 0 4 8}$ \\
\hline
\end{tabular}

Bold represents the significant $P$ values

In this cohort, $73 \%$ of ER+ subtype expressed high $B M I 1$ while $60 \%$ of the ER-negative subtype expressed high BMII.

\section{BMI1 and clinicopathological features}

High BMI1 protein expression showed an association with clinicopathological parameters characteristic of good prognosis including lower histological grade $(P<0.0001)$, more tubule formation $(P=0.004)$, lower mitotic count $(P<0.0001)$, lower nuclear pleomorphism $(P<0.0001)$, lower NPI scores $(P=0.024)$, special tumour type of good prognosis $(P<0.0001)$ and with tumours showing ER $+(P<0.0001)$ and HER $2-$ phenotypes $(P=0.002)$ (Table 1).

Similar findings were identified in the METABRIC cohort. High expression of BMII mRNA was positively associated with good prognostic factors, such as older age
$(P<0.001)$, postmenopausal status $(P<0.001)$, lower grade $(P<0.0001)$, good NPI prognostic group $(P<0.0001)$, tumours of tubular subtype $(P<0.0001)$ and HER2- phenotypes $(P=0.001)$ as shown in Table 1 .

These associations with good prognostic factors were also obtained in the BC Gene Expression Miner database; high expression of BMIl was associated with older age, good prognostic factors, such as good NPI and luminal A subtype $(P<0.0001)$, as shown in supplementary Fig. 2 .

\section{Outcome analysis}

Univariate survival analysis showed that high BMI1 protein expression was significantly associated with longer survival in terms of breast cancer-specific survival (BCSS) in the whole BC cohort $(P=0.02)$ (Fig. 2a). With regard to molecular subtypes, in the luminal ER+ tumours, high expression of BMI1 was significantly associated with BCSS ( $P=0.04$; Fig. 2b) and distant metastasis free survival (DMFS) $(P=0.04$; supplementary Fig. 3$)$. However, in the basal / triple negative subtype (TNBC), high expression of BMI1 was significantly associated with shorter BCSS $(P=0.04)$ (supplementary Fig. 3). In HER2-positive (HER2+) tumours, no significant association between BMI1 and outcome was identified.

The Cox regression model, including age at diagnosis, tumour size, tumour grade and nodal stage, showed that BMI1 as an independent predictor of good prognosis in the whole $\mathrm{BC}$ cohort and in the luminal $\mathrm{ER}+$ patients $(P=0.04$, HR $0.72 ; 95 \% \mathrm{Cl} 0.51-0.99, P=0.04$, and HR $0.69 ; 95 \% \mathrm{Cl}$ $0.48-0.99$, respectively) (Tables 2,3 ) but not in the TNBC subtype. When the multivariate analysis including BMI1 and 

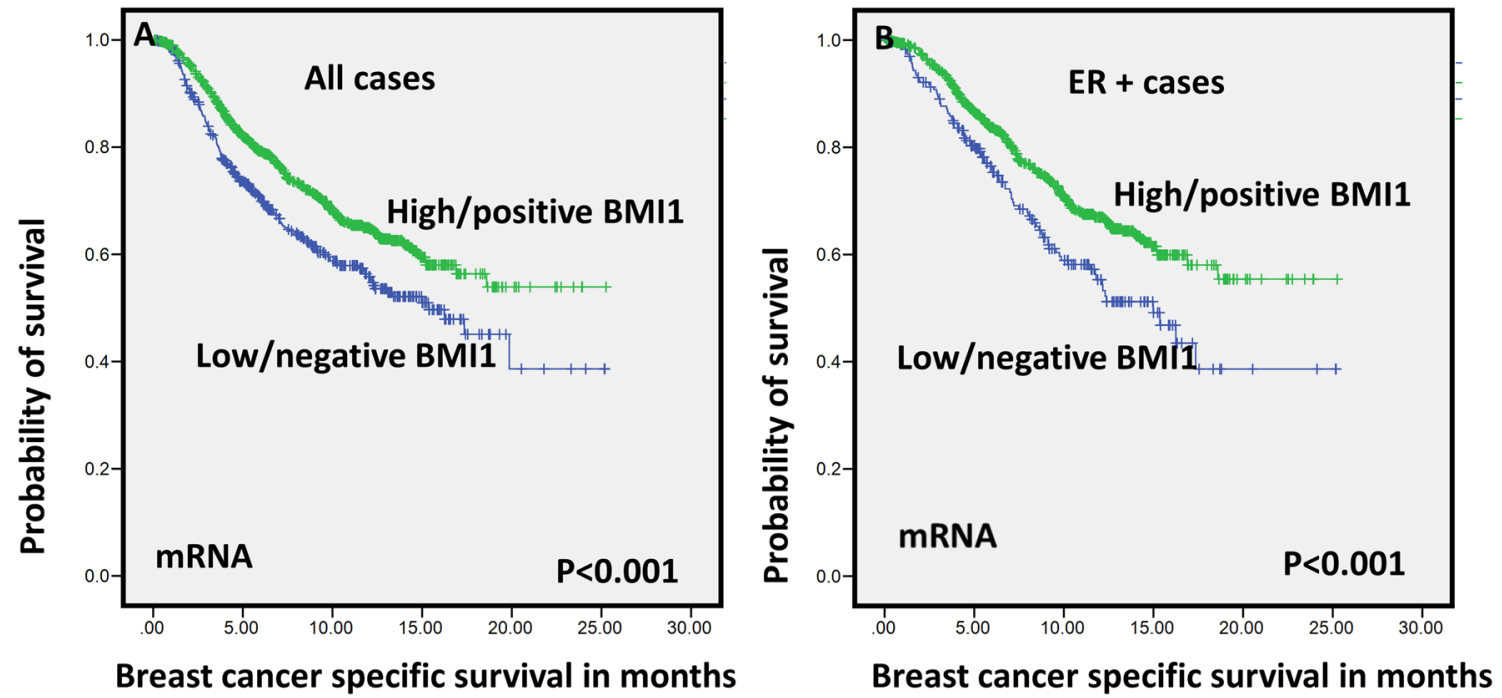

Fig. 3 Kaplan-Meier survival plots for BMI1 expression (mRNA) a breast cancer-specific survival (BCSS) in all cases and b breast cancerspecific survival (BCSS) in ER + cases

Table 3 Multivariate Cox regression hazard model including other prognostic clinicopathological parameters shows that high BMI1 (immunohistochemically) provided an independent prognostic value, associated with longer breast cancer-specific survival in the luminal oestrogen receptor-positive breast cancer

\begin{tabular}{|c|c|c|c|c|}
\hline \multirow[t]{2}{*}{ Variable } & \multirow[t]{2}{*}{ Hazard ratio } & \multicolumn{2}{|c|}{$\begin{array}{l}\text { 95\% Confidence } \\
\text { interval }(\mathrm{CI})\end{array}$} & \multirow[t]{2}{*}{$p$ value } \\
\hline & & Lower & Upper & \\
\hline Patient age & 1.03 & 0.76 & 1.39 & 0.824 \\
\hline Tumour Grade & 1.70 & 1.36 & 2.13 & $<0.0001$ \\
\hline Node stage & 2.03 & 1.62 & 2.56 & $<0.0001$ \\
\hline Tumour size & 0.63 & 0.46 & 0.86 & 0.004 \\
\hline Bmil & 0.69 & 0.48 & 0.99 & 0.047 \\
\hline
\end{tabular}

Bold represents the significant $P$ values

other BCSC (ALDH1A1, CD133, CD24, and SOX9) markers, BMI1 was an independent predictor of good prognosis in the whole $\mathrm{BC}$ cohort $(P=0.017)$, supplementary Table 3 .

With regard to the transcriptomic expression, high $B M I 1$ mRNA expression in the METABRIC cohort was significantly associated with longer BCSS in the whole BC cohort $(P<0.001)$ as well as in the luminal ER+tumours $(P<0.001)$ (see Fig. 3). However, no significant association between $B M I I$ and outcome was observed in ER-tumours or in HER $2+$ patients $(P>0.05)$. Moreover, $\mathrm{CN}$ gain of $B M I 1$ was associated with longer BCSS $(P=0.031)$ in whole BC cohort.

Similar results were observed with the BC Gene Expression Miner dataset, where BMII mRNA was associated with longer survival in the ER+tumour $(P=0.020)$, which confirms the previous findings in the METABRIC cohort as shown in Supplementary Fig. 4.

\section{BMI1 and other biomarkers}

We further investigated the association of BMI1 expression and key BC stem cell (BCSC) biomarkers. At protein level, there was a significant negative correlation between the high expression of BMI1 and BCSC markers: ALDH1A1 $(P=0.017), \mathrm{CD} 133(P=0.006)$ and SOX9 $(P=0.004)$ in all BC cases (supplementary Table $4 \mathrm{a}$ ). In the ER+tumours, BMI1 maintained similar associations and showed negative correlation with ALDH1A1 $(P=0.005)$ and CD133 $(P=0.001)$. Interestingly, in the ER- tumours, BMI1 showed positive association with CD133 $(P<0.0001)$ and SOX10 $(P=0.01)$ expression (supplementary Table $4 \mathrm{a})$.

Similarly at the mRNA level, high expression of $B M I I$ was negatively associated with BCSC including $A L D H 1 A 3$ $(P<0.0001), C D 133(P=0.0003), C D 24(P=0.002)$ and CD44 $(P=0.0007)$ (Supplementary Table $4 b)$ in the whole BC cohort. When the analysis was restricted to the luminal ER+tumours, high BMII expression was associated with low expression of ALDHIAl $(P=0.025), A L D H 1 A 3$ $(P=0.026), C D 133(P=0.038), C D 44(P=0.0001)$, $C D 24(P=0.004)$ and SOX10 (0.0006) (Supplementary Table 4b). In the basal TNBC subtype, positive association was observed between BMII and EPCAM and SOXIO $(P<0.001)$. 


\section{Discussion}

$\mathrm{BC}$ is a heterogeneous disease and the understanding of this heterogeneity is of great importance to improve patient outcome and treatment regimens [30]. Studies have addressed and investigated prognostic factors or biomarkers that illustrate the complex clinical and biological differences in BC subtypes [28, 31, 32]. In the current study, BMI1 expression was evaluated at protein, transcriptomic and genomic levels in well-characterised cohorts of early-stage BC with different molecular subtypes of BC tissue samples.

In the current study, immunopositivity and mRNA of BMI1 were observed in $20 \%$ and $70 \%$ of BC. Previous studies have reported high BMI1 expression in $62 \%$ and $53 \%$ of $\mathrm{BC}[14,33]$. The difference in the frequency of positivity between our results and previous studies could be explained by the difference in the scoring method and varying the definitions of the positivity cut-offs. Our cohort was scored using $\mathrm{H}$-scoring, which is a widely accepted system in both clinical and research settings and the previous studies used other scoring system [17, 34]. BMI1 has been linked with poor prognosis and shorter survival in several cancer types $[35,36]$. However, its prognostic role in $\mathrm{BC}$ remains controversial. Engelsen et al. reported that low expression of BMI1 is associated with loss of ER and PR in endometrial carcinoma [37]. In BC, Kim et al. have reported that BMI1 is associated with positive lymph node metastasis but it was also associated with ER + tumours, which are less aggressive tumour compared with other subtypes of BC [14]. Choi et al. demonstrated that BMI1 is favourable prognostic biomarker in $\mathrm{BC}$ [33].

In this study, a significant correlation between high expression of BMI1 and good prognostic BC features was found in the whole cohort and in the ER+tumours. In ER+ subtype, BMI1 was highly expressed compared to ER-classes. This is in an agreement with Choi et al. study, which showed the same findings and reported that IHC overexpression of BMI1 was associated with ER+expression and other favourable clinicopathological parameters including smaller tumour size, negative lymph node metastasis and intermediate nuclear grade [33].

BMI1 was also here demonstrated to be an independent good prognostic biomarker in ER+tumours, independently of other clinical pathological features [33]. Engelsen et al. have suggested a potential link between BMI1 and hormone receptor status [37]. Interestingly, although there was a limited case number of TNBC subtypes in that study cohort $(n=56)$, increased expression of BMI1 was associated with shorter survival in TNBC [38]. This finding suggests a diverse role for BMI1 in different BC subtypes.

BMI1 plays a vital function in the epigenetic regulation of stem cell transcriptional pathways and is also implicated in the self-renewal, proliferation and cell cycle of CSCs [39, 40]. To the best of our knowledge, this is the first study analysing the association of BMI1 with BCSC biomarkers at both transcriptomic and protein levels. Our data have shown that BMI1 is negatively correlated with some BCSC markers at both mRNA and protein levels in all $\mathrm{BC}$ and the ER+ subtypes; however, the association between BMI1 and BCSC markers in TNBC was limited (only CD133 and SOX10), which further supports a different role of BMI1 in relation to the molecular subtypes of $\mathrm{BC}$ particularly $\mathrm{ER}+$. Our data favour a BCSC function of BMI1 in TNBC but not in ER+ tumours, though this warrants further experimental validation. Our results also showed that the interaction between BMI1 and HER2 is limited and that BMI1 is not associated with outcome in HER2+ tumours.

\section{Conclusion}

The current study indicates that BMI1 exhibited a varied role in BC subtypes. In ER+tumours, BMI1 is associated with good prognosis and longer survival; however, in TNBC, BMI1 showed an association with shorter survival and shows different associations with BCSC markers, which suggest the ER status that may play an important role in modulating the biological function of BMI1. Further functional studies are essential to be performed in order to clarify the significant role of BMI1 in different BC subtypes.

Acknowledgements We thank the University of Nottingham (Nottingham Life Cycle 6) for funding. The authors are part of the PathLAKE digital pathology consortium. These new Centres are supported by a $£ 50 \mathrm{~m}$ investment from the Data to Early Diagnosis and Precision Medicine strand of the government's Industrial Strategy Challenge Fund, managed and delivered by UK Research and Innovation (UKRI).

Data availability The dataset analysed during the current study is available from the corresponding author on reasonable request.

\section{Compliance with ethical standards}

Conflict of interest The authors declare that they have no conflicts of interests.

Ethical approval The experiments comply with the current laws of the country in which they were performed.

Research involving human participants and/or animals This study was approved by the Nottingham Research Ethics Committee 2 under the title 'Development of a molecular genetic classification of breast cancer' and the North West-Greater Manchester Central Research Ethics Committee under the title 'Nottingham Health Science Biobank (NHSB)' reference number 15/NW/0685. All procedures performed in studies involving human participants were in accordance with the ethical standards of the institutional and/or national research committee and with the 1964 Helsinki declaration and its later amendments or comparable ethical standards. Release of data was also pseudoan- 
onymised as per the UK Human Tissue Act regulations. This article does not contain any studies with animals performed by any of the authors.

Informed consent All tissue samples from Nottingham used in this study were pseudoanonymised and collected prior to 1 st September 2006; therefore, under the UK Human Tissue Act, informed patient consent was not needed.

Open Access This article is licensed under a Creative Commons Attribution 4.0 International License, which permits use, sharing, adaptation, distribution and reproduction in any medium or format, as long as you give appropriate credit to the original author(s) and the source, provide a link to the Creative Commons licence, and indicate if changes were made. The images or other third party material in this article are included in the article's Creative Commons licence, unless indicated otherwise in a credit line to the material. If material is not included in the article's Creative Commons licence and your intended use is not permitted by statutory regulation or exceeds the permitted use, you will need to obtain permission directly from the copyright holder. To view a copy of this licence, visit http://creativecommons.org/licenses/by/4.0/.

\section{References}

1. Vaishnave S (2018) BMI1 and PTEN are key determinants of breast cancer therapy: a plausible therapeutic target in breast cancer. Gene 678:302-311

2. Paranjape AN, Balaji SA, Mandal T et al (2014) Bmi1 regulates self-renewal and epithelial to mesenchymal transition in breast cancer cells through Nanog. BMC Cancer 14:785-785

3. Bhattacharya R, Mustafi SB, Street M et al (2015) Bmi-1: at the crossroads of physiological and pathological biology. Genes Dis 2:225-239

4. Cho JH, Dimri M, Dimri GP (2013) A positive feedback loop regulates the expression of polycomb group protein BMI1 via WNT signaling pathway. J Biol Chem 288:3406-3418

5. Liu Y, Liu F, Yu H et al (2012) Akt phosphorylates the transcriptional repressor bmi1 to block its effects on the tumorsuppressing ink4a-arf locus. Sci Signal 5:ra77

6. Kim SH, Singh SV (2015) The role of polycomb group protein Bmi-1 and Notch4 in breast cancer stem cell inhibition by benzyl isothiocyanate. Breast Cancer Res Treat 149:681-692

7. Liu S, Dontu G, Mantle ID et al (2006) Hedgehog signaling and Bmi-1 regulate self-renewal of normal and malignant human mammary stem cells. Cancer Res 66:6063-6071

8. Lee YC, Chang WW, Chen YY et al (2017) Hsp90alpha mediates BMI1 expression in breast cancer stem/progenitor cells through facilitating nuclear translocation of c-Myc and EZH2. Int J Mol Sci 18:1986

9. Wang M-C, Li C-L, Cui J et al (2015) BMI-1, a promising therapeutic target for human cancer. Oncol Lett 10:583-588

10. Guo B-H, Feng Y, Zhang R et al (2011) Bmi-1 promotes invasion and metastasis, and its elevated expression is correlated with an advanced stage of breast cancer. Mol Cancer 10:10

11. Nalwoga H, Arnes JB, Wabinga H, Akslen LA (2010) Expression of aldehyde dehydrogenase 1 (ALDH1) is associated with basal-like markers and features of aggressive tumours in African breast cancer. Br J Cancer 102:369-375

12. Li Y, Wang L, Liu J et al (2017) O-GlcNAcylation modulates Bmi-1 protein stability and potential oncogenic function in prostate cancer. Oncogene 36:6293-6305

13. Li N, Jiang K, Fang LP et al (2018) Knockdown of long noncoding RNA CCAT1 inhibits cell growth, invasion and peritoneal metastasis via downregulation of Bmi-1 in gastric cancer. Neoplasma 65:736-744

14. Kim JH, Yoon SY, Jeong SH et al (2004) Overexpression of Bmi-1 oncoprotein correlates with axillary lymph node metastases in invasive ductal breast cancer. Breast 13:383-388

15. Rakha EA, Pinder SE, Bartlett JM et al (2015) Updated UK Recommendations for HER2 assessment in breast cancer. J Clin Pathol 68:93-99

16. Rakha EA, Agarwal D, Green AR et al (2017) Prognostic stratification of oestrogen receptor-positive HER2-negative lymph node-negative class of breast cancer. Histopathology 70:622-631

17. Green AR, Aleskandarany MA, Agarwal D et al (2016) MYC functions are specific in biological subtypes of breast cancer and confers resistance to endocrine therapy in luminal tumours. $\mathrm{Br}$ J Cancer 114:917-928

18. Hammond ME, Hayes DF, Dowsett M et al (2010) American Society of Clinical Oncology/College of American Pathologists guideline recommendations for immunohistochemical testing of estrogen and progesterone receptors in breast cancer. Arch Pathol Lab Med 134:907-922

19. Althobiti M, Aleskandarany MA, Joseph C et al (2018) Heterogeneity of tumour-infiltrating lymphocytes in breast cancer and its prognostic significance. Histopathology 73:887-896

20. Goldhirsch A, Wood WC, Coates AS et al (2011) Strategies for subtypes-dealing with the diversity of breast cancer: highlights of the St. Gallen International Expert Consensus on the Primary Therapy of Early Breast Cancer 2011. Ann Oncol 22:1736-1747

21. Joseph C, Arshad M, Kurozomi S et al (2018) Overexpression of the cancer stem cell marker CD133 confers a poor prognosis in invasive breast cancer. Breast Cancer Res Treat 174:387

22. Ahmed MA, Aleskandarany MA, Rakha EA et al (2012) A CD44(-)/CD24(+) phenotype is a poor prognostic marker in early invasive breast cancer. Breast Cancer Res Treat 133:979-995

23. Agboola AJ, Paish EC, Rakha EA et al (2012) EpCAM expression is an indicator of recurrence in basal-like breast cancer. Breast Cancer Res Treat 133:575-582

24. Abd El-Rehim DM, Ball G, Pinder SE et al (2005) Highthroughput protein expression analysis using tissue microarray technology of a large well-characterised series identifies biologically distinct classes of breast cancer confirming recent cDNA expression analyses. Int J Cancer 116:340-350

25. McCarty KS Jr, McCarty KS Sr (1984) Histochemical approaches to steroid receptor analyses. Semin Diagn Pathol $1: 297-308$

26. Curtis C, Shah SP, Chin SF et al (2012) The genomic and transcriptomic architecture of 2,000 breast tumours reveals novel subgroups. Nature 486:346-352

27. Pereira B, Chin SF, Rueda OM et al (2016) The somatic mutation profiles of 2,433 breast cancers refines their genomic and transcriptomic landscapes. Nat Commun 7:11479

28. El Ansari R, Craze ML, Miligy I et al (2018) The amino acid transporter SLC7A5 confers a poor prognosis in the highly proliferative breast cancer subtypes and is a key therapeutic target in luminal B tumours. Breast Cancer Res 20:21

29. Jezequel P, Campone M, Gouraud W et al (2012) bc-GenExMiner: an easy-to-use online platform for gene prognostic analyses in breast cancer. Breast Cancer Res Treat 131:765-775

30. Januskeviciene I, Petrikaite V (2019) Heterogeneity of breast cancer: the importance of interaction between different tumor cell populations. Life Sci 239:117009

31. Aleskandarany MA, Sonbul S, Surridge R et al (2017) RhoGTPase activating-protein 18: a biomarker associated with good prognosis in invasive breast cancer. Br J Cancer 117:1176-1184 
32. Toss MS, Miligy IM, Gorringe KL et al (2018) Prolyl-4-hydroxylase Alpha subunit 2 (P4HA2) expression is a predictor of poor outcome in breast ductal carcinoma in situ (DCIS). Br J Cancer 119:1518-1526

33. Choi YJ, Choi YL, Cho EY et al (2009) Expression of Bmi-1 protein in tumor tissues is associated with favorable prognosis in breast cancer patients. Breast Cancer Res Treat 113:83-93

34. Arora A, Abdel-Fatah TM, Agarwal D et al (2016) Clinicopathological and prognostic significance of RECQL5 helicase expression in breast cancers. Carcinogenesis 37:63-71

35. Glinsky GV, Berezovska O, Glinskii AB (2005) Microarray analysis identifies a death-from-cancer signature predicting therapy failure in patients with multiple types of cancer. J Clin Invest 115:1503-1521

36. Song LB, Zeng MS, Liao WT et al (2006) Bmi-1 is a novel molecular marker of nasopharyngeal carcinoma progression and immortalizes primary human nasopharyngeal epithelial cells. Cancer Res 66:6225-6232

37. Engelsen IB, Mannelqvist M, Stefansson IM et al (2008) Low BMI-1 expression is associated with an activated BMI-1-driven signature, vascular invasion, and hormone receptor loss in endometrial carcinoma. Br J Cancer 98:1662-1669

38. Wang Y, Zhe H, Ding Z et al (2012) Cancer stem cell marker Bmi-1 expression is associated with basal-like phenotype and poor survival in breast cancer. World J Surg 36:1189-1194

39. Lessard J, Sauvageau G (2003) Bmi-1 determines the proliferative capacity of normal and leukaemic stem cells. Nature 423:255-260

40. Park IK, Qian D, Kiel M et al (2003) Bmi-1 is required for maintenance of adult self-renewing haematopoietic stem cells. Nature 423:302-305

Publisher's Note Springer Nature remains neutral with regard to jurisdictional claims in published maps and institutional affiliations. 